\title{
Thermodynamic Study of the Interaction of Bovine Serum Albumin and Amino Acids with Cellulose Nanocrystals
}

\author{
Salvatore Lombardo, ${ }^{\dagger}$ Samuel Eyley, ${ }^{*}{ }^{\dagger}$ Christina Schütz, ${ }^{\dagger}$ Hans van Gorp, ${ }^{\S}$ Sabine Rosenfeldt, ${ }^{\prime \prime}$ \\ Guy Van den Mooter, ${ }^{\ddagger}$ and Wim Thielemans* ${ }^{* \dagger}$
}

\begin{abstract}
${ }^{\dagger}$ Renewable Materials and Nanotechnology Research Group, Department of Chemical Engineering, KU Leuven, Campus Kulak Kortrijk, Etienne Sabbelaan 53 box 7659, 8500 Kortrijk, Belgium

${ }^{*}$ Drug Delivery and Disposition, Department of Pharmaceutical and Pharmacological Sciences, KU Leuven, O \& N II, Herestraat 49 box 921, 3000 Leuven, Belgium

${ }^{\S}$ Division of Molecular Imaging and Photonics, Department of Chemistry, KU Leuven Celestijnenlaan, 200 F, 3001 Leuven, Belgium

"Physical Chemistry I and Bavarian Polymer Institute, University Bayreuth, Universitätsstrasse 30, 95440 Bayreuth, Germany
\end{abstract}

\section{Supporting Information}

ABSTRACT: The interaction of bovine serum albumin (BSA) with sulfated, carboxylated, and pyridinium-grafted cellulose nanocrystals (CNCs) was studied as a function of the degree of substitution by determining the adsorption isotherm and by directly measuring the thermodynamics of interaction. The adsorption of BSA onto positively charged pyridinium-grafted cellulose nanocrystals followed Langmuirian adsorption with the maximum amount of adsorbed protein increasing linearly with increasing degree of substitution. The binding mechanism between the positively charged pyridinum-grafted cellulose nanocrystals and BSA was found to be endothermic and based on charge neutralization. A positive entropy of adsorption associated with an increase of the degree of disorder upon addition of

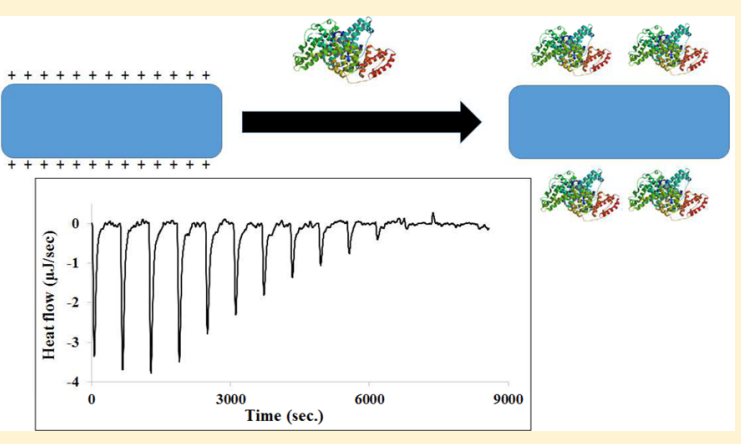
BSA compensated for the unfavorable endothermic enthalpy and enabled formation of pyridinium-g-CNC-BSA complexes. The endothermic enthalpy of adsorption was further found to decrease as a function of increasing degree of substitution. Negatively charged cellulose nanocrystals bearing sulfate and/or carboxylic functionalities were found to not interact significantly with the BSA protein. To investigate in more detail the role of single amino acids in the adsorption of proteins onto cellulose nanocrystals, we also studied the interaction of different types of amino acids with CNCs, i.e., charged (lysine, aspartic acid), aromatic (tryptophan, tyrosine), and polar (serine) amino acids. We found that none of the single amino acids bound with CNCs irrespective of surface charge and that therefore the binding of proteins with CNCs appears to require larger amino acid sequences that induce a greater entropic contribution to stabilize binding. Single amino acids are thus not adsorbed onto cellulose nanocrystals.

\section{INTRODUCTION}

The self-assembly of nanoparticles into ordered structures has already been investigated widely to control the fabrication of materials and devices with new or improved optical, electronic, magnetic, mechanical, or active properties. ${ }^{1}$ Controlling this self-assembly process is paramount for the reproducible production of materials with consistent properties, and is thus a large focus of current research efforts. ${ }^{2}$ A more fundamental understanding of the thermodynamics of the interactions at play is crucial to develop the insights needed to exert the necessary control over these self-assembly processes. For this purpose, isothermal titration calorimetry (ITC) is a promising technique. It can be used to directly probe the thermodynamic interactions in real time during nanoparticle assembly. Pioneering studies were reported in 2004, in which ITC was employed to characterize the energetics of interaction between gold nanoparticles with biological ligands such as amino acids ${ }^{3}$ and DNA. ${ }^{4}$ However, the use of this technique in nanotechnology is still in its infancy, with few documented studies in the literature to date. ${ }^{5}$ One reason may be that this technique experiences many challenges and more detailed studies are required to make ITC a more common technique in nanotechnology. The main difficulty comes from the fact that heat is a global parameter; therefore, impurities or secondary processes can also give rise to heat exchange, which could cover or alter the real heat of interaction. Therefore, high purity of the samples and high control over the system under investigation is a major requirement.

In this work, we used ITC to investigate the interaction of the protein bovine serum albumin (BSA) and different amino

Received: March 2, 2017

Revised: April 12, 2017

Published: May 11, 2017 
acids with cellulose nanocrystals (CNCs). Rod-shaped cellulose nanocrystals are attractive for new material development because of their abundance, renewability, and physicochemical properties such as high surface area, low density, mechanical strength, and potentially low production cost. ${ }^{6-8}$ A variety of possible applications have been reported, such as reinforcing agents, optical materials, electronics supports, paint additives and stabilizers, and security papers. ${ }^{7,9,10}$ Rod-shaped CNCs with variable dimensions can be obtained from different cellulose sources such as cotton, wood, and tunicates through a controlled hydrolysis with inorganic acids. When using sulfuric acid, the CNC particles are stabilized in aqueous suspension through the repulsion of the deprotonated sulfate ester groups, which are grafted onto the surface of the nanoparticles during the acid hydrolysis. ${ }^{11}$

Sulfated CNCs self-assemble in water to form chiral nematic structures. ${ }^{12}$ In these structures, the cellulose nanoparticles are organized in left-handed helicoids with pitch values typically around $1-2 \mu \mathrm{m}$ (just above the reflection wavelength of visible light). ${ }^{6,13}$ This pitch can be reduced by increasing the concentration of CNCs, ${ }^{6}$ increasing the ionic strength, ${ }^{14}$ addition of glucose, ${ }^{13}$ and ultrasonication. ${ }^{15}$ Expanding on these ideas further, changing the environment through the addition of other nanoparticles, proteins, polymers, or small molecules will also alter the interactions between CNCs. Understanding how these interactions can be manipulated is crucial to generate ordered structures, and thus macroscopic functional materials, in a controlled manner. ${ }^{2}$ Another way to tailor the interactions between nanoparticles and of nanoparticles with its environment is by altering their surface functionalities. Virtually any functional groups can be inserted on the surface of cellulose nanocrystals. ${ }^{16}$ There is also interest in controlling nanocellulose interactions with biological systems for their applications as, for example, drug delivery carriers. ${ }^{17}$ To this effect, in vivo studies have already highlighted the biocompatibility of CNCs in mice. ${ }^{18}$ However, the use of cellulose nanocrystals as drug carriers or other agents in biological systems requires a fundamental understanding of the interactions of cellulose nanoparticles with both simple and complex biomolecules. Guo et al. investigated the thermodynamics and the specificity of the binding of cellulose nanocrystals with a cellulose binding module. ${ }^{19}$ This same group also identified and characterized the minimum peptide sequence composed of seven residues which was able to bind cellulose nanocrystals, while also studying the thermodynamics of the process using ITC. ${ }^{20}$

A better understanding of the interactions between nanocellulose and biomolecules is also needed to improve enzymatic degradation processes, which are important for the use of CNCs in commercial applications. Recently, it has been shown that the addition of bovine serum albumin (BSA) improves the enzymatic degradation of cellulose. ${ }^{21}$ Formation of a so-called protein corona (protein adsorption layer) in a biological medium formed by protein-nanoparticle interactions has not been reported for CNCs, while it has been widely studied in BSA-gold nanoparticle systems. ${ }^{22,23}$ As the interaction of nanoparticles with living matter is dictated by the protein corona, it is important to understand its formation and structure in much more detail.

The adsorption of BSA onto 10 different cellulose derivatives could be described by Langmuirian adsorption and was found to depend on $\mathrm{pH}$, ionic strength, and surface charge. ${ }^{24}$ In addition, Lavenson et al. used magnetic resonance imaging combined with UV-spectroscopy to investigate the adsorption of BSA onto four different cellulose fibers, showing that only a small amount of protein was adsorbed. More specifically, the authors showed a decrease in the free protein concentration of $\sim 5 \%$; however, the authors state that this value is smaller than the error range of the technique employed (10\%). ${ }^{25}$ This was later confirmed by surface plasmon resonance measurements published by Orelma et al., who also highlighted that a charge difference between BSA and cellulose would increase the adsorption efficiency dramatically. ${ }^{26}$ In addition, Taajamaa et al. showed that BSA does not adsorb on pure cellulose. ${ }^{27}$ Finally, Zimnitsky et al. then investigated the adsorption of various amino acids onto carboxymethyl cellulose fibers, showing multilayer adsorption on oxidized cellulose at a high concentration of amino acids in the treatment medium. ${ }^{28}$ The thermodynamics of the adsorption of BSA onto CNCs has not yet been reported in the literature and could help to explain some of the apparently conflicting results mentioned above. The dependence of the interactions as a function of the amount of surface functionality has also not yet been described.

In this work, we have studied the interactions of BSA and single amino acids with cellulose nanocrystals. To investigate the effect of surface characteristics, we used sulfated, carboxylated, and pyridinium-grafted cellulose nanocrystals with different degrees of substitution. We also investigated the effect of the addition of free amino acids into the CNC suspension to measure interactions between single residues and cellulose nanocrystals.

\section{EXPERIMENTAL SECTION}

Materials and Methods. Cotton wool, hydrochloric acid (37\%, extra pure), pyridine (99\%, for synthesis), sodium hydroxide (99\%, p.a., ISO, in pellets), dichloromethane (99.5\%, for synthesis), Ltryptophan (>98.5\%), sodium hypochlorite (12\%), and sodium bromide were purchased from Carl Roth. Bovine serum albumin (>96\%) and L-lysine (>98\%) were purchased from Sigma-Aldrich. Sulfuric acid (95\%), pyridine (AnalaR NORMAPUR, 99.7\%), methanol (99.8\%), and ethanol (absolute) were purchased from VWR international. $p$-Toluenesulfonyl chloride (98\%), 4-(1bromoethyl)benzoic acid (98\%), and 4-(bromomethyl)benzoic acid (97\%) were purchased from Alfa Aesar. L-Tyrosine was purchased from TCI, and (2,2,6,6-tetramethylpiperidin-1-yl)oxyl (TEMPO), Laspartic acid (>98\%), and L-serine were purchased from Acros Organics.

Synthesis and purification of sulfated cellulose nanocrystals were done by standard sulfuric acid hydrolysis as previously described. ${ }^{29}$ Sulfated CNCs were used as starting materials for further surface functionalization. Cationic pyridinium-grafted CNCs were synthesized using a previously described one-pot reaction, using 4-(1-bromoethyl/ bromomethyl)benzoic acid and 4-toluenesulfonyl chloride as reagents with pyridine as a solvent. ${ }^{30}$ Different degrees of substitution were obtained by varying reaction temperature, time, and stoichiometry of reagents (see the Supporting Information for details). Carboxylated cellulose nanocrystals were prepared via oxidation with $(2,2,6,6-$ tetramethylpiperidin-1-yl)oxyl (TEMPO), as previously described. ${ }^{31}$ The procedures used for the preparation of the different CNCs employed in this work are described in detail in the Supporting Information. All of the nanocrystals used in this work were fully characterized by methods developed in our laboratory which employed various techniques. ${ }^{32}$ Infrared spectroscopy (IR) was used to verify that the modifications were performed successfully; elemental analysis (EA) was used to determine the amount of modification; thermogravimetric analysis (TGA) was used to estimate the water content; X-ray photoelectron spectroscopy (XPS) was employed to measure the elemental composition on the surface of the nanoparticles; X-ray diffraction (XRD) was employed to determine the 
crystallinity of the CNCs; and a detailed morphological analysis was performed using atomic force microscopy (AFM) for the determination of the cellulose nanoparticle lengths and small angle $\mathrm{X}$-ray scattering (SAXS) was used to determine the width. Full characterization data are reported in the Supporting Information.

Adsorption Isotherm Experiments. Binding constants describing the adsorption of BSA onto cellulose nanocrystals at $25{ }^{\circ} \mathrm{C}$ were determined from adsorption isotherms. All measurements were carried out in glass vials containing serial dilutions of BSA mixed with an equal volume of an aqueous suspension of CNC to a final cellulose concentration of $1-2 \mathrm{mg} / \mathrm{mL}$. All adsorption experiments were carried out at least in duplicate. Both positively charged (pyridinium-grafted) and negatively charged (sulfated or carboxylated) cellulose nanocrystals were used. Each suspension was also stirred for at least $15 \mathrm{~min}$ before measurement. Previously reported work investigating CNCprotein interactions used centrifugation to remove $\mathrm{CNC}$-bound proteins quantitatively. ${ }^{19}$ In this work, we used filtration with a 0.2 $\mu \mathrm{M}$ syringe-filter to retain the CNCs, with or without bound BSA, as tests showed that this method resulted in a more effective separation than could be achieved with centrifugation. The filtrate containing unbound BSA was measured spectrophotometrically at $280 \mathrm{~nm}$ using a Shimadzu UV-1800 spectrophotometer. The equilibrium association constant $\left(K_{\mathrm{a}}\right)$ describing the adsorption of BSA onto cellulose nanocrystals was determined by nonlinear regression of bound versus free protein concentrations to a one-binding-site Langmuir model. ${ }^{19}$ The equation used was the following

$$
B=\frac{B_{\max } K_{\mathrm{a}} F}{1+\left(F \times K_{\mathrm{a}}\right)}
$$

where $F$ is the concentration of free protein, determined from the maximum absorption at $280 \mathrm{~nm} ; B$ is the concentration of bound protein per gram of cellulose, calculated by subtracting $F$ from the starting protein concentration; and $B_{\max }$ is the maximum amount of adsorbed protein, determined from the experiment. These experiments were also carried out for the aromatic amino acids tryptophan and tyrosine, as they can be detected in solution by UV-vis spectroscopy.

Isothermal Titration Calorimetry. Calorimetric experiments were performed using a TAM III isothermal calorimeter (TA Instruments). The experiments were carried out at neutral $\mathrm{pH}$ in aqueous solution. In order to minimize the $\mathrm{pH}$ change during the experiments, the protein was dialyzed against Milli- $\mathrm{Q}$ water, and the dialysate was used to suspend the nanoparticles and to dilute the protein solutions. The measured $\mathrm{pH}$ of BSA was in the range 7-7.5. All samples were thoroughly degassed by sonicating for $15 \mathrm{~min}$ under a vacuum before the experiments. The system was equilibrated at 25 ${ }^{\circ} \mathrm{C}$ while stirring at $200 \mathrm{rpm}$ until the baseline shift was $<50 \mathrm{nW} / \mathrm{h}$. Titrations were performed by injecting the solution of protein or amino acid from the syringe into the ITC sample cell containing cellulose substrate suspensions, allowing 10 min between injections. The protein concentration in the injection solution was confirmed spectrophotometrically at $280 \mathrm{~nm}$. A blank experiment to estimate the heat of dilution was also performed at $25{ }^{\circ} \mathrm{C}$ using identical injections of protein solution into the dialysate, and all calorimetric traces were corrected for the heat of dilution by subtracting the blank from test experiments. At least two independent titration experiments were performed to confirm consistency. A thermodynamic profile of binding interaction was determined by fitting the data to an independent binding site interaction model as described by Freyer and Lewis. ${ }^{33}$ Nonlinear regression was used along with the model equations to determine the best values of the fitting parameters (e.g. $K_{a}, \Delta H$, and $n$ ). The ITC thermogram for a generic binding process is modeled by the expression relative to the equilibrium constant (eq 2) and the mass balance (eq 3 ) equations

$$
\Theta_{j}=\frac{[\mathrm{L}] K_{j}}{1+[\mathrm{L}] K_{j}}
$$

$$
L_{\mathrm{t}}=[\mathrm{L}]+P_{\mathrm{t}} \sum_{j=1}^{k}\left(n_{j} \Theta_{j}\right)
$$

where $\Theta_{j}$ is the fraction of site $j$ occupied by the ligand, $L_{t}$ is the total ligand concentration, $[\mathrm{L}]$ is the free ligand concentration, $P_{\mathrm{t}}$ is the total macromolecule concentration, $K_{j}$ is the binding constant for process $j$, and $n_{j}$ is the total stoichiometric ratio for process $j$. The fraction of occupied binding sites $j$ can be calculated substituting $[\mathrm{L}]$ into eq 2 .

The total heat produced can be calculated from eq 4 , where $V_{0}$ is the initial volume of the sample cell and $\Delta H_{j}$ is the molar enthalpy change for process $j$. The differential heat is defined by eq 5 :

$$
\begin{aligned}
& Q=P_{\mathrm{t}} V_{0} \sum_{j=1}^{k}\left(n_{j} \Theta_{j} \Delta H_{j}\right) \\
& \Delta Q=Q(i)-Q(i-1)
\end{aligned}
$$

Nonlinear regression was performed on the parameters $K_{\mathrm{a}}$ (assuming a single adsorption process), $n$, and $\Delta H$ to obtain a best fit to the experimental data, using NanoAnalyze data analysis software (TA Instruments). The Gibbs free energy change $\Delta G$ was calculated from the association constant using eq 6 and the change in entropy $\Delta S$ using eq 7 :

$$
\begin{aligned}
& \Delta G=-R T \ln \left(K_{\mathrm{a}}\right) \\
& \Delta G=\Delta H-T \Delta S
\end{aligned}
$$

Determination of Bound Amino Acid by Acid-Base Titrations. To determine the amount of amino acid bound to $\mathrm{CNCs}$, a solution of charged amino acid was mixed with a suspension of the nanocrystals. The suspension was stirred using a magnetic stirrer and then filtered to remove the nanocrystals and bound amino acid. The amount of free amino acid was determined by acid-base titration using a SI analytics Titroline 6000 autotitrator.

\section{RESULTS AND DISCUSSION}

Adsorption Isotherm Experiments. The adsorption of BSA was first studied by adsorption experiments by measuring the free BSA concentration via UV-spectroscopy. Cellulose nanocrystals with different degrees of substitution of pyridinium grafts were used.

BSA is adsorbed strongly onto pyridinium-grafted cellulose nanocrystals, and the behavior was described using the standard Langmuir model (Figure 1). The association constant was determined by fitting the adsorption plots using eq 1 . The

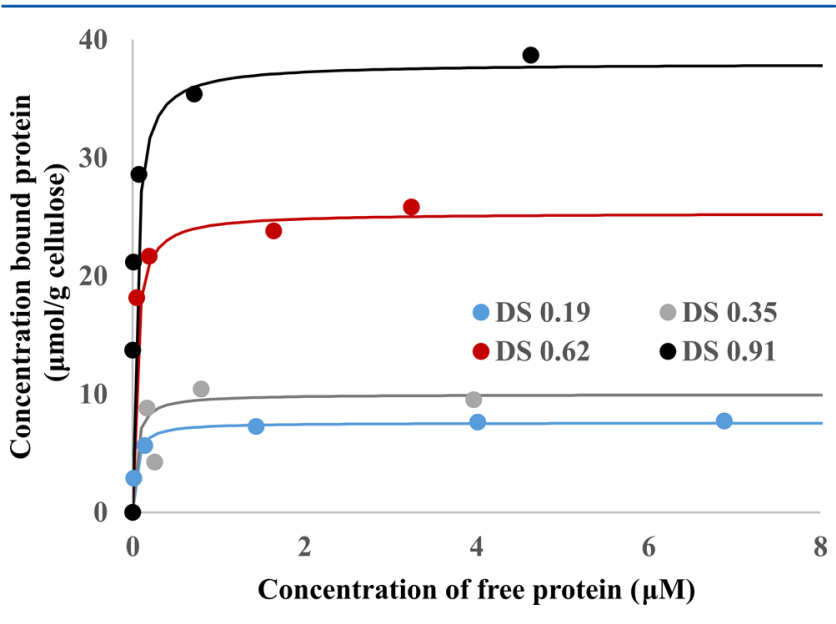

Figure 1. Adsorption of BSA in $0.1 \%$ pyridinium-grafted nanocrystals and fit according to eq 1. Pyridinium-grafted nanocrystals with four different degrees of substitution were used. 


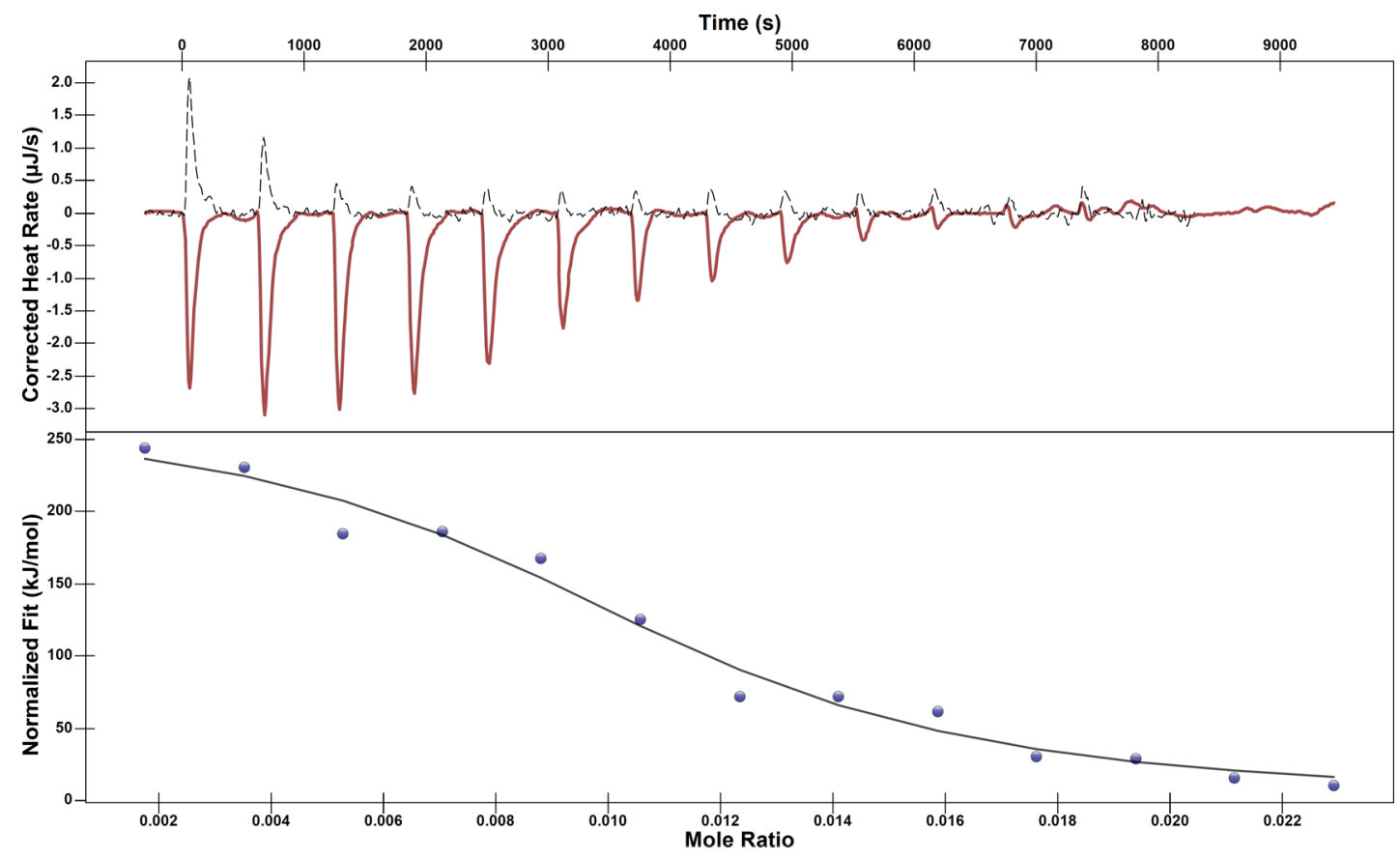

Figure 2. ITC data for the adsorption of BSA onto pyridinium-grafted nanocrystals. In the top graph, the solid red line shows the calorimetric trace obtained from the addition of $0.1 \mathrm{mM}$ BSA to a suspension containing $1.6 \mathrm{mg} / \mathrm{mL}$ of pyridinium-grafted CNC $(\mathrm{DS}=0.62)$ and the dotted black line shows the calorimetric trace obtained from the dilution of BSA. Each peak corresponds to a $15 \mu \mathrm{L}$ addition. The bottom graph shows the heat adsorbed after every addition of protein determined from the integral of the corresponding peak, which was plotted as a function of the molar ratio ( $\mathrm{mol}$ of $\mathrm{BSA} / \mathrm{mol}$ of pyridinium groups).

Table 1. Values of $B_{\max }$ and $K_{\mathrm{a}}$ for the Adsorption of BSA onto Pyridinium-Grafted Nanocrystals Determined from the ITC and UV-Spectroscopy ${ }^{a}$

$\begin{array}{ccccc}\begin{array}{c}\text { degree of substitution } \\ \text { (a.u.) }\end{array} & \begin{array}{c}B_{\max }(\mu \mathrm{mol} / \mathrm{g} \text { of cellulose) from } \\ \text { ITC }\end{array} & \begin{array}{c}B_{\max }(\mu \mathrm{mol} / \mathrm{g} \text { of cellulose }) \\ \text { spectroscopy }\end{array} & \begin{array}{c}K_{\mathrm{a}}\left(\mu \mathrm{M}^{-1}\right) \text { from } \mathrm{UV}- \\ \text { ITC }\end{array} & \begin{array}{c}K_{\mathrm{a}}\left(\mu \mathrm{M}^{-1}\right) \text { from UV- } \\ \text { spectroscopy }\end{array} \\ 0.19 \pm 0.01 & 2.5 \pm 0.4 & 7.8 \pm 0.2 & 7 \pm 5 & 31 \pm 5 \\ 0.35 \pm 0.01 & 4.0 \pm 0.6 & 8 \pm 2 & 2 \pm 7 & 27 \pm 9 \\ 0.62 \pm 0.01 & 8 \pm 1 & 22 \pm 4 & 1.0 \pm 0.5 & 10 \pm 5 \\ 0.91 \pm 0.03 & 12.7 \pm 0.7 & 34 \pm 3 & & 8 \pm 6 \\ { }^{a} \text { Nanocrystals with four different degrees of substitution were used. } & & \end{array}$

values of the association constant determined from the fits as a function of the degree of substitution were found to decrease linearly with increasing degree of substitution of the CNCs (Figure $3 \mathrm{~b}$ ). This can be explained by taking into account the steric hindrance for the adsorbate which is higher for nanocrystals with a higher grafting density, in line with published studies which show weaker interactions for the adsorption of polymers of different sizes onto carbon ${ }^{34}$ and gold substrates. ${ }^{35,36}$ The maximum amount of protein adsorbed $\left(B_{\max }\right)$ however increased with the increasing degree of substitution (Figure 3a). Therefore, the increase of steric hindrance does not seem to prevent the protein from adsorbing onto the modified cellulose nanocrystals. Instead, this dependence suggests that the difference of charge between modified cellulose nanocrystals and BSA has a dominant effect on the value of $B_{\max }$. The observed trend is in line with literature data, where the amount of adsorbed protein was found to be indeed dependent on the surface charge. ${ }^{26}$ The amount adsorbed was saturated for all batches at an equilibrium concentration of BSA $<0.5 \mu \mathrm{M}$. This value is more than 10 -fold lower than values reported for the adsorption of BSA onto a series of other cellulose derivatives and shows that the specificity of BSA toward cellulose-grafted nanocrystals is higher than the specificity of BSA toward a series of cellulose derivatives reported in the literature. ${ }^{24}$ In addition, the values of $K_{\mathrm{a}}$ for the adsorption of BSA onto pyridinium-grafted cellulose nanocrystals are $\sim 100$-fold higher than the association constant determined for the adsorption of the cellulose binding module determined using the same technique, while the $B_{\max }$ values are in the same range. ${ }^{19}$ As shown in Figure 3b, there is a significant error on the values of the determined association constant, due to the low concentration of BSA present at the equilibrium point before saturation. Such a low amount makes it more difficult to accurately determine the value of $K_{a}$ as the absorbance of the peak at $280 \mathrm{~nm}$ is close to the detection limit. Our results obtained also showed a higher $K_{\mathrm{a}}$ than literature values relative to the adsorption of various ligands onto cellulose. This is most likely due to the different cellulose derivatives employed in the literature and the different conditions used. ${ }^{24,26,25}$ Adsorption experiments performed using the same method showed that negatively charged cellulose (sulfated and carboxylated CNCs) did not interact significantly with negatively charged BSA. The absorption at $280 \mathrm{~nm}$ obtained from the filtered mixture of BSA and negative cellulose could be completely superimposed onto the absorption peak obtained from a BSA solution with the same 

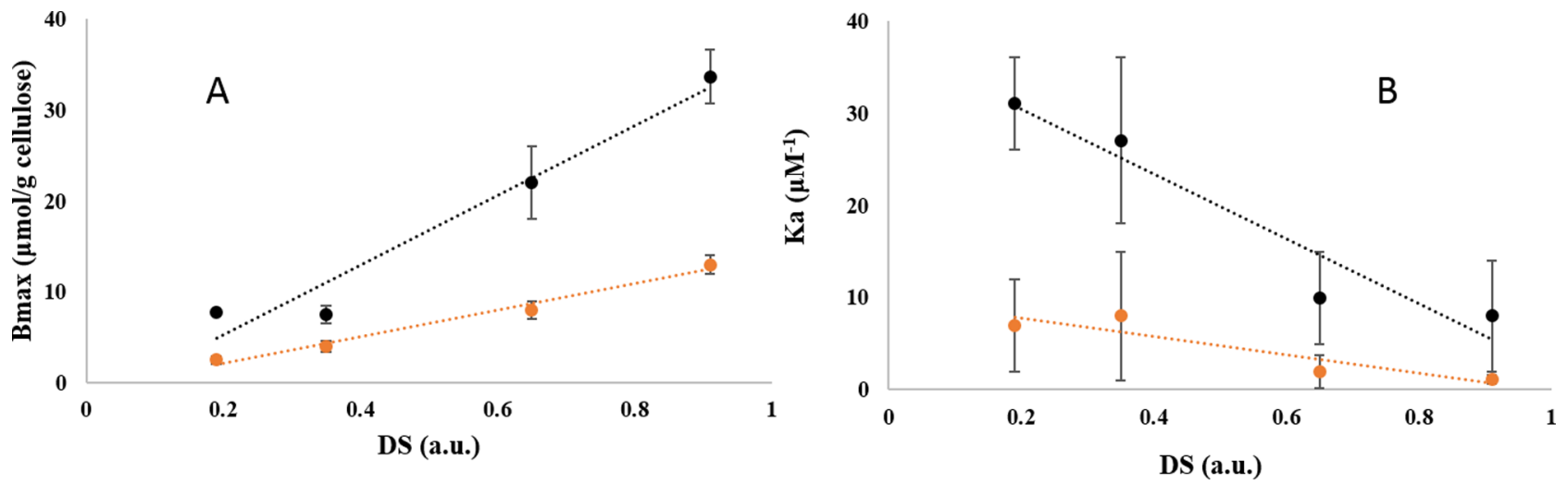

Figure 3. (A) Maximum adsorption of BSA onto pyridinium-grafted cellulose nanocrystals as a function of the degree of substitution determined by UV-spectroscopy (black points) and by ITC (orange points). (B) Association constant as a function of the degree of substitution determined by UVspectroscopy (black points) and by ITC (orange points).

protein concentration. Those results agree with the literature, which shows very little to no adsorption of BSA onto negatively charged nanocellulose and cellulose fibers ${ }^{25,26}$ and no adsorption on pure cellulose. ${ }^{27}$

Isothermal Titration Calorimetry. As binding of BSA was only seen for positively charged CNCs, we investigated the binding of BSA onto the pyridinium-grafted cellulose nanocrystals with four different degrees of substitution by ITC. The calorimetric traces yielded a positive energy deflection, indicating endothermic binding. Figure 2 shows the calorimetric trace obtained as a result of the addition of $0.1 \mathrm{mM}$ BSA to a suspension of $1.6 \mathrm{mg} / \mathrm{mL}$ of pyridinium-grafted $\mathrm{CNCs}$ (representative for all the other measurements) as well as the model fit. The model parameters for the model fits are combined in Table 1.

ITC showed that the association constant decreased linearly as the degree of substitution increased (Figure $3 \mathrm{~b}$ ), in line with UV-spectroscopy measurements. However, the values of $K_{\mathrm{a}}$ obtained by ITC were lower than the values obtained using UV-spectroscopy. This difference can be associated with the high error associated with the association constant determined by UV-spectroscopy as mentioned before. The maximum BSA adsorption value increased linearly with an increasing degree of substitution (Figure 3a), similarly to the values determined by UV-spectroscopy. However, the values obtained by ITC are again lower by approximately a factor of 2 . We associate the difference between the results obtained from the two techniques with the separation method employed in the spectrophotometric experiments. In fact, some of the smallest particles pass through the $0.2 \mu \mathrm{m}$ filter, which is expected considering the size distribution determined by AFM (see Supporting Information, Table S1.12). Such particles absorb in the UV region, which is expected due to the aromatic nature of the graft, giving an error in the measurements. In order to reduce this error, we used a solution of a filtered suspension as a reference. However, when BSA is bound, the length and the width of the nanocrystals increase, as indicated by the results obtained using AFM and SAXS (see Supporting Information, Table S1.12). As the size increases, a higher number of particles is retained by the filter. The increase of the size of the nanocrystals would decrease the value of absorbance at $280 \mathrm{~nm}$, since less particles are present in the filtrate, therefore giving an underestimation of the measured value of free protein concentration. This explains also the increased difference in $B_{\max }$ at higher DS. In fact, at higher DS, the particles will have a higher absorbance value, because of higher numbers of aromatic grafts attached. This also explains the different slope of the straight lines describing the behavior of both $B_{\max }$ and $K_{\mathrm{a}}$ as a function of the degree of substitution, as CNCs with a higher number of aromatic grafts would absorb more, therefore increasing the error in the UV measurements.

Overall, the results obtained using this model are comparable (Table 1 ), as $B_{\max }$ determined by ITC is only slightly lower than the values obtained using UV-spectroscopy. The fitted $K_{\mathrm{a}}$ values obtained calorimetrically on the other hand are found to be about 5-fold lower than the results determined by UVspectroscopy. Both techniques also showed the same experimental trend for both parameters as a function of the degree of substitution.

The thermodynamics of the binding process was then elucidated from the integral plot of the calorimetric traces. The thermodynamic values measured relative to the adsorption of BSA onto pyridinium-grafted cellulose are shown in Table 2,

Table 2. Thermodynamics Parameters Obtained for the Binding of BSA on Pyridinium-Grafted Cellulose Nanocrystals

$\begin{array}{cccc}\text { degree of substitution (a.u.) } & \Delta H(\mathrm{~kJ} / \mathrm{mol}) & \Delta S(\mathrm{~J} / \mathrm{mol} \cdot \mathrm{K}) & \Delta G(\mathrm{~kJ} / \mathrm{mol}) \\ 0.19 \pm 0.01 & 228 \pm 10 & 890 \pm 40 & -37 \pm 2 \\ 0.35 \pm 0.01 & 211 \pm 9 & 830 \pm 30 & -36 \pm 3 \\ 0.62 \pm 0.01 & 274 \pm 11 & 1040 \pm 30 & -35 \pm 1 \\ 0.91 \pm 0.03 & 280 \pm 3 & 1050 \pm 10 & -33 \pm 1\end{array}$

and plotted in Figure 4, as a function of the degree of substitution. The thermodynamic parameters determined show that the process is entropically driven, since the positive value of entropy more than compensates for the positive enthalpy contribution to the Gibbs free energy, yielding an overall negative value of the Gibbs free energy. A positive value of both enthalpy and entropy is usually characteristic of the loss of preferential polar interactions (commonly incorrectly referred to as hydrophobic interactions), ${ }^{37-39}$ and can be attributed to the collapse of the structure of water molecules surrounding the hydrophilic CNC rods. ${ }^{40}$ This is supported by the observation of precipitation occurring after the addition of BSA to saturate the suspension of pyridinium-grafted nanocellulose. The occurrence of precipitation was also observed by visual inspection in all products inside the calorimetric cells after the experiments. In order to further investigate the precipitation 

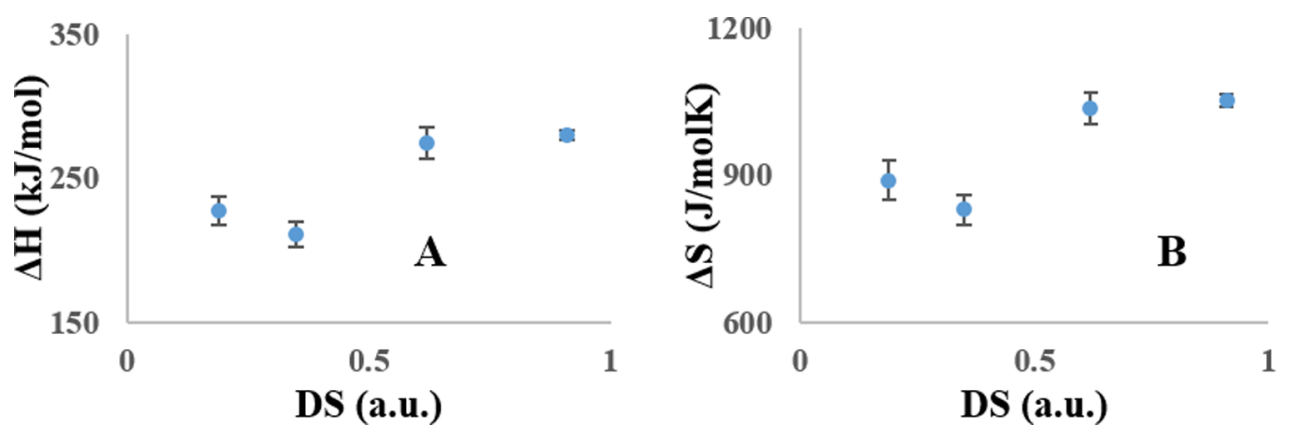

Figure 4. Thermodynamic parameters determined as a function of the degree of substitution. Panel A shows the enthalpy values obtained. Calculated entropy values are shown in panel B.

of nanocrystals, experiments were performed using a glass vial, consisting of the addition of aliquots of BSA to a suspension of pyridinium-grafted cellulose nanocrystals. The suspension started to precipitate at a protein concentration close to the saturation point.

Data shown in Figure 4 indicate that both the enthalpy and entropy increased with increasing degree of substitution. The increase in entropy can be associated with an increase in the disorder inside the suspension, occurring when a higher amount of protein is adsorbed onto the surface of the cellulose rods, thereby releasing surface-structured water molecules present before adsorption. The decrease in the Gibbs free energy as a function of the degree of substitution is directly linked with the decrease in the association constant with an increased number of grafts (eq 6). This trend can be explained by taking into account the steric hindrance which is higher in nanocrystals with a higher grafting density. Indeed, at higher grafting densities, it is more likely that a bound protein will cover more than one binding site than at lower grafting densities. If we consider the crystal structure of $\mathrm{CNCs}^{41}$ we can estimate a surface area per graft of ca. $0.23 \mathrm{~nm}^{2}$ for the highest DS and $1.1 \mathrm{~nm}^{2}$ for the lowest DS. Comparing the values of surface area per graft with the dimension of BSA determined from its crystal structure, ${ }^{42}$ we find that the protein in its folded structure is too big to occupy a single site (details of calculations can be found in Supporting Information S5). Furthermore, we determined a molar ratio of BSA/pyridinium grafts of $\sim 0.01$ at the equivalence point (Figure 2, bottom graph) irrespective of the DS of pyridinium groups on the CNCs. This means that a single protein always occupies approximately 100 pyridinium groups, and will thus be more spread out over the CNC surface at lower DS. This could be associated with a conformational change of the protein, which can spread upon binding, thus losing its folded structure. This spreading upon adsorption will obviously become more difficult as more proteins attempt to adsorb at higher DS, giving rise to the measured steric hindrance effect, and leading to a more dense protein structure. With this knowledge, it then becomes possible to control the BSA protein structure at the CNC surface by simply choosing the correct DS. In addition looking at the primary structure of BSA, ${ }^{42}$ we observed that there are 99 acidic amino acids (aspartic acid and glutamic acid), which agrees with the number of pyridinium groups occupied by a single BSA molecule. From this consideration, we predicted that the acidic residues are involved in the adsorption of BSA on positively charged CNCs. It should be pointed out that only a small part of the acidic amino acids are expected to be deprotonated at the investigated $\mathrm{pH}$. In fact, the $\mathrm{pH}$ of the BSA solution was in the range 7-7.5, higher than the isoelectric point of the protein $(\mathrm{pI}=5.86)$, giving an expected net charge between -14 and $-20 .^{43}$ Therefore, if binding occurs at all 100 sites, the release of protons from the glutamate and aspartate residues in BSA is to be expected during binding. To verify this hypothesis, we performed $\mathrm{pH}$ experiments to follow the change of $\mathrm{pH}$ associated with the process. To be able to detect $\mathrm{pH}$ changes, we used a higher concentration of CNCs and BSA compared to the ITC experiments and we observed indeed a decrease in the $\mathrm{pH}$ linked to the proton release (see Supporting Information, Figure S4.5).

Investigation of Interaction between CNC and Single Amino Acids. In order to understand if the binding between BSA or other proteins and cellulose nanocrystals was due to single residues or a more global structure, we also investigated the interaction between single amino acids and cellulose nanocrystals. For aromatic amino acids (tryptophan and tyrosine), the peaks at $280 \mathrm{~nm}$ obtained from a solution of the pure molecule and from the filtered mixture of amino acid with modified or sulfated cellulose were superimposable, indicating that there is no discernible binding. This was verified by ITC, from the linear trend observed, with $\Delta H_{\text {app }}=-5 \pm 1$ $\mathrm{kJ} / \mathrm{mol}$ (see Supporting Information, Figure S2.12). Similarly, we also found that the mixing of serine with sulfated CNCs gave a linear trend, with $\Delta H_{\text {app }}=-1.7 \pm 0.3 \mathrm{~kJ} / \mathrm{mol}$ (see Supporting Information, Figure S2.11). The enthalpy values measured for both amino acids are in line with reported values for the heat of protonation of the carboxylic group. ${ }^{44}$ It is plausible to assume that a large fraction of the carboxylates are protonated if we consider the $\mathrm{pH}$ change of both amino acids occurring during the titration, going from 7.1-7.7 (above the amino acid's isoelectric point, $\left.\mathrm{pI}_{\text {trp }}=5.89, \mathrm{pI}_{\mathrm{ser}}=5.68\right),{ }^{45}$ to $\sim 3.1$ (below the isoelectric point) (see Supporting Information, Figure S4.6). The addition of lysine to sulfated cellulose nanocrystals gave rise to a high exothermic heat $(\Delta H=-70.7$ $\pm 0.1 \mathrm{~kJ} / \mathrm{mol}$ ), with the heat exchange as a function of the molar ratio showing a sigmoidally shaped curve (see Figure 5). This behavior was confirmed by $\mathrm{pH}$ titration where the titration of sulfated $\mathrm{CNCs}$ with lysine showed a similar sigmoidally shaped curve, with a comparable stoichiometric number $(n \approx$ 1) (see Supporting Information, Figure S4.1). For the sulfated CNC-lysine interaction, both enthalpy and stoichiometric number were dependent on the starting $\mathrm{pH}$ of the suspension. In fact, when the calorimetric titrations were performed by adding lysine to a sulfated $\mathrm{CNC}$ suspension neutralized to $\mathrm{pH}$ 7 , the enthalpy of interaction decreased to $-47 \pm 2 \mathrm{~kJ} / \mathrm{mol}$, which was comparable to the enthalpy of the dilution of lysine in water $(\Delta H=-43 \pm 2 \mathrm{~kJ} / \mathrm{mol})$, and also comparable to the 


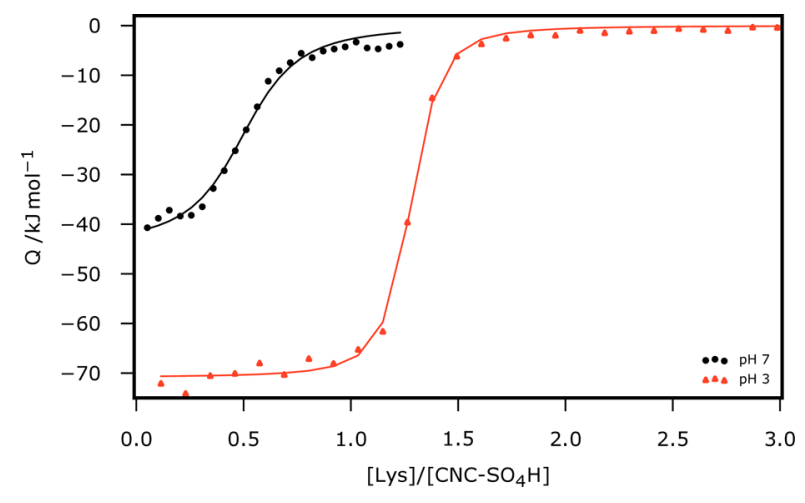

Figure 5. Integral plot obtained from additions of $5 \mu \mathrm{L}$ of $5 \mathrm{mM}$ lysine to sulfated $\mathrm{CNCs}$ at acid $\mathrm{pH}$ (orange triangles) and at neutral $\mathrm{pH}$ (black circles).

enthalpy of protonation of the amino groups reported for various amino acids. ${ }^{44}$ Looking into the binding of lysine to sulfated and carboxylated CNCs in more detail using acid-base titration, we observed no discernible binding, since the titration curve obtained from the filtered mixture of lysine-CNCs was superimposable with the curve obtained from a solution of the same concentration of lysine (see Supporting Information, Figures S4.2 and S4.3). This indicates that the interaction between lysine and negatively charged CNCs is mainly due to a straightforward acid-base equilibrium. In addition, we investigated the binding of aspartic acid by acid-base titration and ITC, to confirm the role of negatively charged amino acids in the adsorption of BSA onto pyridinium-grafted CNCs. Results show that the electrostatic interaction between aspartic acid and pyridinium-grafted CNCs was not strong enough for the CNCs to retain the amino acid on the filter (see Supporting Information, Figure S2.6 for ITC and Figure S4.4 for acid-base titration). Taking together all of these results, it appears that single amino acids are not capable of binding with cellulose nanocrystals, and that a larger supramolecular structure with more synergistic binding characteristics is required for strong binding. These results agree with recently reported data, which show that a minimum amount of seven amino acids is required to bind with cellulose nanocrystals. ${ }^{20}$ Zimnitsky et al. ${ }^{28}$ also stated that adsorption of amino acids occurred only when highly concentrated solutions of amino acids (up to $2.7 \mathrm{M}$ ) were used. These conditions are very different from the dilute conditions we employed in our work where there is no overruling concentration driving force toward adsorption.

\section{CONCLUSIONS}

The binding of BSA to cellulose nanocrystals was dependent on the charge displayed on the cellulose nanocrystals. Negatively charged CNCs (sulfated and carboxylated) did not show significant BSA-CNC binding. Positively charged (pyridiniumgrafted) CNCs on the other hand showed adsorption dependent on the charge density on the CNC surface. The latter adsorption followed the Langmuir adsorption model, with the amount of protein adsorbed on the cellulose particles proportional to the degree of substitution of positive pyridinium grafts. The association constant decreased with the number of positively charged groups attached on the surface of cellulose nanocrystals. This was linked with the increase of the steric hindrance generated from the higher amount of protein adsorbed. BSA was also found to alter its structure to retain the same amount of pyridinium groups with which to interact, i.e., $\sim 100$. Thermodynamically, the calorimetric data showed that entropy is the main driving force for the adsorption process. The positive entropy was associated with an increase of the degree of disorder upon addition of BSA, compensating for an unfavorable endothermic enthalpy. The value of entropy increased as a function of the degree of substitution, indicating that the disorder of the suspension increased as the total amount of protein adsorbed increased. The positive value of entropy was associated with the collapse of the structure of water molecules surrounding the hydrophilic cellulose rods, as confirmed by the precipitation of the suspended particles. The entropy was also found to increase as a function of the degree of substitution, linked with an increase in hydrophobicity as the total amount of protein adsorbed increased. The increase of hydrophobicity is also related to the increase in size of the particles, occurring when the protein is adsorbed onto the surface. The different binding behavior of BSA to negatively and positively charged CNCs is proof of the crucial role of the charge difference between cellulose nanocrystals and proteins to modulate protein adsorption. This agrees with recently published data, where the crucial role of the charge difference between nanocellulose and BSA in the adsorption process was also highlighted. ${ }^{26}$ The authors were also able to detect a very small amount of negative protein adsorbed onto negative cellulose nanocrystals using surface plasmon resonance. This inconsistency with our work is caused either from the better detection of the surface plasmon resonance (SPR) compared to UV-spectroscopy or from the ability of SPR to use larger concentrations of BSA in the streaming solution which would saturate UV detection. Furthermore, during SPR measurements, a constant flow of protein solution is supplied over an immobilized cellulose matrix, pushing the protein to adsorb, whereas we investigated the adsorption in suspension, where this constant driving force is not supplied.

We finally also investigated the interaction between single amino acids and cellulose, to understand if single residues are capable of adsorption onto CNCs, as this has not yet been reported for positively charged CNCs. We showed that interactions arising from acid-base equilibria are dominant and that single amino acids do not bind to cellulose, independent of the charge of both the amino acid and the cellulose nanocrystals. This is in agreement with previously published data that used phage display to characterize binding on negatively charged CNCs. ${ }^{20}$ Our findings thus demonstrate that a proper three-dimensional structure is necessary to allow single residues to bind to cellulose nanocrystals.

\section{ASSOCIATED CONTENT}

\section{S Supporting Information}

The Supporting Information is available free of charge on the ACS Publications website at DOI: 10.1021/acs.langmuir.7b00710.

Additional information on the synthesis and characterization of cellulose nanocrystals, isothermal titration calorimetry, UV-spectroscopy, acid-base titrations, and determination of the surface area per graft (PDF)

\section{AUTHOR INFORMATION}

\section{Corresponding Authors}

*E-mail: samuel.eyley@kuleuven.be.

*E-mail: wim.thielemans@kuleuven.be. 


\section{ORCID}

Wim Thielemans: 0000-0003-4451-1964

\section{Notes}

The authors declare no competing financial interest.

\section{ACKNOWLEDGMENTS}

The authors thank Paul Mack and Christopher Deeks at Thermo Fisher Scientific East Grinstead XPS applications laboratory (East Grinstead, U.K.) for performing XPS measurements for this work. Financial support for this work was provided by Research Foundation-Flanders (FWO) under the Odysseus program grant G.0C60.13 $\mathrm{N}$ and research grant $1501516 \mathrm{~N}, \mathrm{KU}$ Leuven (grant OT/14/072), and the Province of West-Vlaanderen - Belgium (W.T.'s Provincial Chair in Advanced Materials).

\section{REFERENCES}

(1) Yi, G.-R.; Pine, D. J.; Sacanna, S. Recent Progress on Patchy Colloids and Their Self-Assembly. J. Phys.: Condens. Matter 2013, 25 (19), 193101.

(2) Grzelczak, M.; Vermant, J.; Furst, E. M.; Liz-Marzán, L. M. Directed Self-Assembly of Nanoparticles. ACS Nano 2010, 4 (7), 3591-3605.

(3) Joshi, H.; Shirude, P. S.; Bansal, V.; Ganesh, K. N.; Sastry, M. Isothermal Titration Calorimetry Studies on the Binding of Amino Acids to Gold Nanoparticles. J. Phys. Chem. B 2004, 108 (31), 1153511540 .

(4) Gourishankar, A.; Shukla, S.; Ganesh, K. N.; Sastry, M. Isothermal Titration Calorimetry Studies on the Binding of DNA Bases and PNA Base Monomers to Gold Nanoparticles. J. Am. Chem. Soc. 2004, 126 (41), 13186-13187.

(5) Limo, M. J.; Perry, C. C.; Thyparambol, A. A., Wei, Y.; Latour, R. A. Bio-Inspired Nanotechnology: From Surface Analysis to Applications. In Bio-Inspired Nanotechnology: From Surface Analysis to Applications; Knecht, M. R., Walsh, T. R., Eds.; Springer Science +Business Media: New York, 2014; pp 37-94.

(6) Lagerwall, J. P. F.; Schütz, C.; Salajkova, M.; Noh, J.; Hyun Park, J.; Scalia, G.; Bergström, L. Cellulose Nanocrystal-Based Materials: From Liquid Crystal Self-Assembly and Glass Formation to Multifunctional Thin Films. NPG Asia Mater. 2014, 6 (1), e80.

(7) Eichhorn, S. J.; Dufresne, A.; Aranguren, M.; Marcovich, N. E.; Capadona, J. R.; Rowan, S. J.; Weder, C.; Thielemans, W.; Roman, M.; Renneckar, S.; et al. Review: Current International Research into Cellulose Nanofibres and Nanocomposites. J. Mater. Sci. 2010, 45 (1), $1-33$.

(8) Kelly, J. A.; Giese, M.; Shopsowitz, K. E.; Hamad, W. Y.; MacLachlan, M. J. The Development of Chiral Nematic Mesoporous Materials. Acc. Chem. Res. 2014, 47 (4), 1088-1096.

(9) Eyley, S.; Shariki, S.; Dale, S. E. C.; Bending, S.; Marken, F.; Thielemans, W. Ferrocene-Decorated Nanocrystalline Cellulose with Charge Carrier Mobility. Langmuir 2012, 28 (16), 6514-6519.

(10) Dumanli, A. G.; Kamita, G.; Landman, J.; van der Kooij, H.; Glover, B. J.; Baumberg, J. J.; Steiner, U.; Vignolini, S. Controlled, BioInspired Self-Assembly of Cellulose-Based Chiral Reflectors. Adv. Opt. Mater. 2014, 2 (7), 646-650.

(11) Habibi, Y.; Lucia, L. A.; Rojas, O. J. Cellulose Nanocrystals: Chemistry, Self-Assembly, and Applications. Chem. Rev. 2010, 110, 3479-3500.

(12) Revol, J. F.; Bradford, H.; Giasson, J.; Marchessault, R. H.; Gray, D. G. Helicoidal Self-Ordering of Cellulose Microfibrils in Aqueous Suspension. Int. J. Biol. Macromol. 1992, 14 (3), 170-172.

(13) Mu, X.; Gray, D. G. Formation of Chiral Nematic Films from Cellulose Nanocrystal Suspensions Is a Two-Stage Process. Langmuir 2014, 30 (31), 9256-9260.

(14) Beck-Candanedo, S.; Roman, M.; Gray, D. G. Effect of Reaction Conditions on the Properties and Behavior of Wood Cellulose Nanocrystal Suspensions. Biomacromolecules 2005, 6 (2), 1048-1054.
(15) Beck, S.; Bouchard, J.; Berry, R. Controlling the Reflection Wavelength of Iridescent Solid Films of Nanocrystalline Cellulose. Biomacromolecules 2011, 12 (1), 167-172.

(16) Eyley, S.; Thielemans, W. Surface Modification of Cellulose Nanocrystals. Nanoscale 2014, 6 (14), 7764-7779.

(17) Lohcharoenkal, W.; Wang, L.; Chen, Y. C.; Rojanasakul, Y. Protein Nanoparticles as Drug Delivery Carriers for Cancer Therapy. BioMed Res. Int. 2014, 2014, 180549.

(18) Colombo, L.; Zoia, L.; Violatto, M. B.; Previdi, S.; Talamini, L.; Sitia, L.; Nicotra, F.; Orlandi, M.; Salmona, M.; Recordati, C.; et al. Organ Distribution and Bone Tropism of Cellulose Nano-Crystals in Living Mice. Biomacromolecules 2015, 16 (9), 2862-2871.

(19) Guo, J.; Catchmark, J. M. Binding Specificity and Thermodynamics of Cellulose-Binding Modules from Trichoderma Reesei Cel7A and Cel6A. Biomacromolecules 2013, 14 (5), 1268-1277.

(20) Guo, J.; Catchmark, J. M.; Mohamed, M. N. A.; Benesi, A. J.; Tien, M.; Kao, T. H.; Watts, H. D.; Kubicki, J. D. Identification and Characterization of a Cellulose Binding Heptapeptide Revealed by Phage Display. Biomacromolecules 2013, 14 (6), 1795-1805.

(21) Wang, H.; Mochidzuki, K.; Kobayashi, S.; Hiraide, H.; Wang, X.; Cui, Z. Effect of Bovine Serum Albumin (BSA) on Enzymatic Cellulose Hydrolysis. Appl. Biochem. Biotechnol. 2013, 170 (3), 541551.

(22) Cedervall, T.; Lynch, I.; Lindman, S.; Berggård, T.; Thulin, E.; Nilsson, H.; Dawson, K. A.; Linse, S. Understanding the NanoparticleProtein Corona Using Methods to Quantify Exchange Rates and Affinities of Proteins for Nanoparticles. Proc. Natl. Acad. Sci. U. S. A. 2007, 104 (7), 2050-2055.

(23) Lynch, I.; Dawson, K. A. Protein-Nanoparticle Interactions. Nano Today 2008, 3 (1-2), 40-47.

(24) Ha, K.-S.; Hinago, H.; Sakoda, A.; Suzuki, M. Adsorption of Bovine Serum Albumin onto Surface-Modified Carbon Fibers and Cellulose Derivatives. Stud. Surf. Sci. Catal. 1993, 80, 251-258.

(25) Lavenson, D. M.; Tozzi, E. J.; McCarthy, M. J.; Powell, R. L.; Jeoh, T. Investigating Adsorption of Bovine Serum Albumin on Cellulosic Substrates Using Magnetic Resonance Imaging. Cellulose 2011, 18 (6), 1543-1554.

(26) Orelma, H.; Filpponen, I.; Johansson, L. S.; Laine, J.; Rojas, O. J. Modification of Cellulose Films by Adsorption of $\mathrm{Cmc}$ and Chitosan for Controlled Attachment of Biomolecules. Biomacromolecules 2011, 12 (12), 4311-4318.

(27) Taajamaa, L.; Rojas, O. J.; Laine, J.; Yliniemi, K.; Kontturi, E. Protein-Assisted 2D Assembly of Gold Nanoparticles on a Polysaccharide Surface. Chem. Commun. 2013, 49, 1318-1320.

(28) Zimnitsky, D. S.; Yurkshtovich, T. L.; Bychkovsky, P. M. Multilayer Adsorption of Amino Acids on Oxidized Cellulose. J. Colloid Interface Sci. 2005, 285 (2), 502-508.

(29) Jasmani, L.; Eyley, S.; Van Gorp, H.; Schütz, C.; De Feyter, S.; Thielemans, W. One-Pot Functionalization of Cellulose Nanocrystals with Various Cationic Groups. Cellulose 2016, 23, 3569-3576.

(30) Jasmani, L.; Eyley, S.; Wallbridge, R.; Thielemans, W. A Facile One-Pot Route to Cationic Cellulose Nanocrystals. Nanoscale 2013, 5 (21), 10207-10211.

(31) Habibi, Y.; Chanzy, H.; Vignon, M. R. TEMPO-Mediated Surface Oxidation of Cellulose Whiskers. Cellulose 2006, 13 (6), 679687.

(32) Eyley, S.; Schütz, C.; Thielemans, W. Surface Chemistry and Characterization of Cellulose Nanocrystals. In Advances in Cellulose Science and Technology: Chemistry, Analysis, and Applications; Rosenau, T., Potthast, A., Eds.; John Wiley \& Sons, Inc: Hoboken, NJ, USA, 2017.

(33) Freyer, M. W.; Lewis, E. a. Isothermal Titration Calorimetry: Experimental Design, Data Analysis, and Probing Macromolecule/ Ligand Binding and Kinetic Interactions. Methods Cell Biol. 2008, 84, 79-113.

(34) Shokri, R.; Vonau, F.; Cranney, M.; Aubel, D.; Narladkar, A.; Isare, B.; Bouteiller, L.; Simon, L.; Reiter, G. Consequences of Varying Adsorption Strength and Adding Steric Hindrance on Self-Assembly of 
Supramolecular Polymers on Carbon Substrates. J. Phys. Chem. C 2012, 116, 21594-21600.

(35) Vonau, F.; Suhr, D.; Aubel, D.; Bouteiller, L.; Reiter, G.; Simon, L. Evolution of Multilevel Order in Supramolecular Assemblies. Phys. Rev. Lett. 2005, 94, 066103.

(36) Vonau, F.; Linares, M.; Isare, B.; Aubel, D.; Habar, M.; Bouteiller, L.; Reiter, G.; Geskin, V.; Zerbetto, F.; Lazzaroni, R.; et al. Branched Substituents Generate Improved Supramolecular Ordering in Physisorbed Molecular Assemblies. J. Phys. Chem. C 2009, 113, 4955-4959.

(37) Haq, I.; Ladbury, J. E.; Chowdhry, B. Z.; Jenkins, T. C.; Chaires, J. B. Specific Binding of Hoechst 33258 to the $\mathrm{d}$ (CGCAAATTTGCG)2 Duplex: Calorimetric and Spectroscopic Studies. J. Mol. Biol. 1997, 271 (2), 244-257.

(38) Privalov, P. L.; Gill, S. J. The Hydrophobic Effect: A Reappraisal. Pure Appl. Chem. 1989, 61 (6), 1097-1104.

(39) Silverstein, K. A. T.; Haymet, A. D. J.; Dill, K. A. A Simple Model of Water and the Hydrophobic Effect. J. Am. Chem. Soc. 1998, 120 (13), 3166-3175.

(40) Chiad, K.; Stelzig, S. H.; Gropeanu, R.; Weil, T.; Klapper, M.; Müllen, K. Isothermal Titration Calorimetry: A Powerful Technique To Quantify Interactions in Polymer Hybrid Systems. Macromolecules 2009, 42 (19), 7545-7552.

(41) Nishiyama, Y.; Sugiyama, J.; Chanzy, H.; Langan, P. Crystal Structure and Hydrogen Bonding System in Cellulose $\mathrm{I} \alpha$ from Synchrotron X-Ray and Neutron Fiber Diffraction. J. Am. Chem. Soc. 2003, 125 (47), 14300-14306.

(42) Majorek, K. A.; Porebski, P. J.; Dayal, A.; Zimmerman, M. D.; Jablonska, K.; Stewart, A. J.; Chruszcz, M.; Minor, W. Structural and Immunologic Characterization of Bovine, Horse, and Rabbit Serum Albumins. Mol. Immunol. 2012, 52 (3-4), 174-182.

(43) Medda, L.; Barse, B.; Cugia, F.; Bostro, M.; Parsons, D. F.; Ninham, B. W.; Monduzzi, M.; Salis, A. Hofmeister Challenges: Ion Binding and Charge of the BSA Protein as Explicit Examples. Langmuir 2012, 28, 16355-16363.

(44) Sovago, I.; Kiss, T.; Gergely, A. Critical Survey of the Stability Constants of Complexes of Aliphatic Amino Acids. Pure Appl. Chem. 1993, 65 (5), 1029-1080.

(45) Lide, D. R. Dissociation Constants of Organic Acids and Bases. CRC Handbook of Chemistry and Physics; CRC Press: Boca Raton, FL, USA, 2005; pp 1276-1286. 